\title{
Rematching AGS Booster synchrotron injection lattice for smaller transverse beam emittances
}

\author{
C. Liu, J. Beebe-Wang, K. Brown, \\ C. Gardner, H. Huang, M. Minty, \\ V. Schoefer, K. Zeno
}

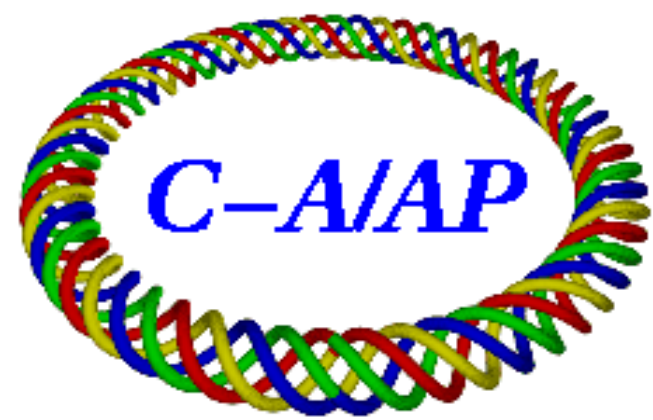

Collider-Accelerator Department Brookhaven National Laboratory Upton, NY 11973

U.S. Department of Energy Office of Science, Office of Nuclear Physics

Notice: This document has been authorized by employees of Brookhaven Science Associates, LLC under Contract No. DE-SC0012704 with the U.S. Department of Energy. The United States Government retains a nonexclusive, paid-up, irrevocable, world-wide license to publish or reproduce the published form of this document, or allow others to do so, for United States Government purposes. 


\title{
REMATCHING AGS BOOSTER SYNCHROTRON INJECTION LATTICE FOR SMALLER TRANSVERSE BEAM EMITTANCES
}

\author{
C. Liu, J. Beebe-Wang, K. Brown, C. Gardner, H. Huang, M. Minty, V. Schoefer, K. Zeno, \\ Brookhaven National Lab, Upton, NY, USA.
}

\section{Abstract}

The polarized proton beam is injected into the booster via the charge-exchange (H- to $\mathrm{H}+)$ scheme. The emittance growth due to scattering at the stripping foil is proportional to the beta functions at the foil. It was demonstrated that the current scheme of reducing the beta functions at the stripping foil preserves the emittance better; however the betatron tunes are above but very close to half integer. Due to concern of space charge and half integer in general, options of lattice designs aimed towards reducing the beta functions at the stripping foil with tunes at more favorable places are explored.

\section{INTRODUCTION}

The Booster [1] is a circular accelerator with a circumference of about $200 \mathrm{~m}$, one forth that of AGS synchrotron. It receives polarized proton beam from $200 \mathrm{MeV}$ Linac and ion beams from EBIS or tandem. There are 24 FODO cells with separate function magnets, organized into six super-periods with two missing dipoles in each super-period in order to accommodate spaces needed for RF acceleration, injection, extraction and abort systems. The overall layout of the Booster ring is shown in Figure 1, where the super-periods are designated as A through F. The polarized proton beam is injected at C5 magnet, the stripping foil is after C5 and before C6 quadrupole. The nominal Twiss parameters of the Booster ring are shown in Figure 2.

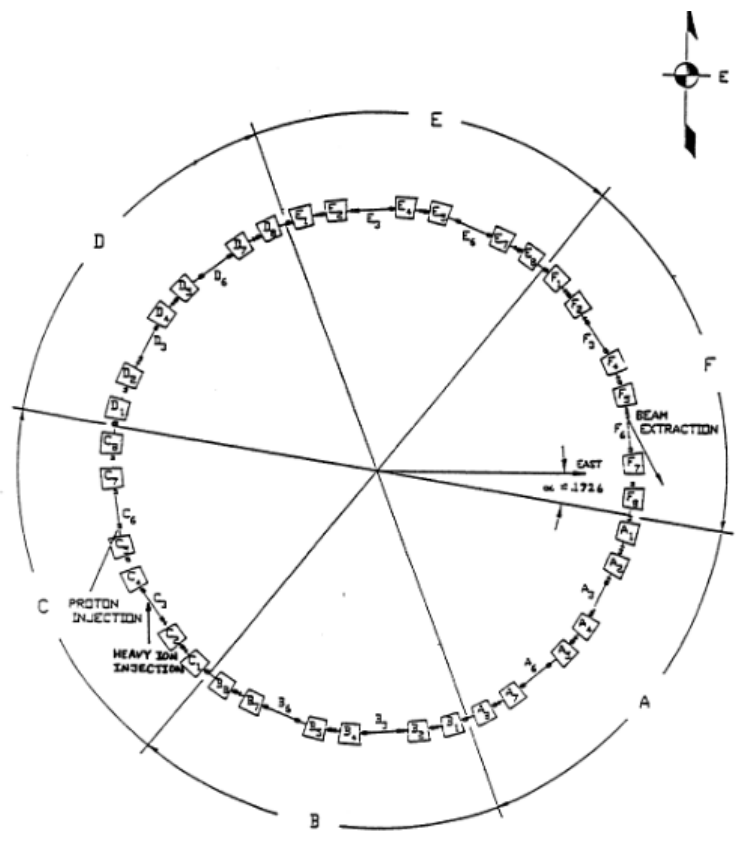

Figure 1: The overall layout of the Booster ring with 6 super-periods. 


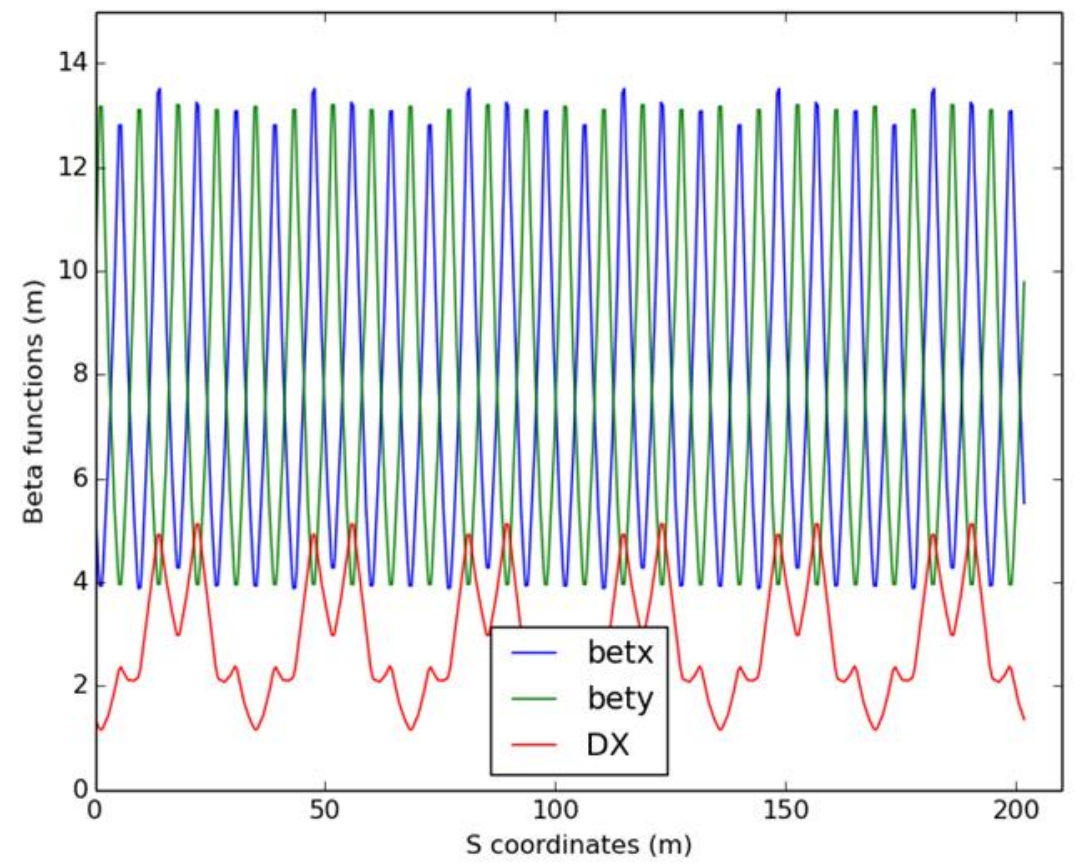

Figure 2: The Twiss parameters of the Booster ring, the horizontal beta function in blue, the vertical beta function in green and the horizontal dispersion in red.

Each quadrupole has a main winding consisting of 5 turns, tune winding of 1 turn and an auxiliary (or trim winding) consisting of 2 turns. The auxiliary windings are used for the half-integer correction. The wiring scheme of the half integer quadrupole is listed in reference [2].

The multiple scattering on the stripping foil is one of the contributors to the emittance growth in the Booster. The emittance growth due to multiple scattering [3] can be approximated by

$$
\Delta \epsilon_{r m s}=\frac{1}{2} n \beta(s) \theta_{r m s}^{2}
$$

where $\mathrm{n}$ is the number of traversals through the foil and depends on the linac pulse length and shape of the foil, $\beta(\mathrm{s})$ is the matched $\beta$-function at the foil, and $\theta$ is the rms multiple scattering per turn, which depends on the foil hickness and material. In this report, the focus is on the scheme of reducing the beta functions at the stripping foil for better preserved beam emittance.

The current scheme of reducing beta functions at the stripping foil is reported in [3]. The betatron tunes are brought close to and above half integer, and then the lattice is distorted by exciting the half integer stopband quadrupole strings. It was demonstrated that the horizontal beam emittance was better preserved with reduced beta functions in this scheme, while the effect in the vertical plane is not obvious.

Even though whether space charge is contributing to the emittance growth remains as a question, it is desirable to reduce the beta functions without getting tunes close to 0.5 . This report presents alternative schemes to reduce the beta functions at the foil while keep the tunes at more favorable places.

\section{ALTERNATIVE SOLUTION WITH HIGHER BETATRON TUNES}

In the MAD-X simulation, the quadrupoles close to the foil are chosen as variables. Various numbers of quadrupoles were tested. It was assumed that additional power supplies will be put on the main windings of selected 
quads. The required currents from the additional power supplies are $\sim 200 \mathrm{~A}$. There is concern of voltage overload on these power supplies during Booster ramping [4].

We also tried putting additional power supplies on the tune windings of selected quads. The $1 / 2$ integer power supplies are employed in the optimization as well with the current limit $(-20,20 \mathrm{~A})$ [4]. The required currents from the additional power supplies are $\sim 400 \mathrm{~A}$. This option is not practical because the required current on the tune windings are too high.

The half integer resonance strings were employed as variables. The tune strings were used to compensate the resulted tune changes. The tunes were held at 4.7/4.75. The required currents for the resonance strings are: qvstr $1=$ 29.5; qvstr2 $=-73.0$; qhstr $1=-62.1$; qhstr2 $=-74.6$. The required current for tune strings are: iqhe $=79.2$; iqvc $=$ 94.3. The achieved beta functions at the foil are $(6,3) \mathrm{m}$ as shown in Figure 3.

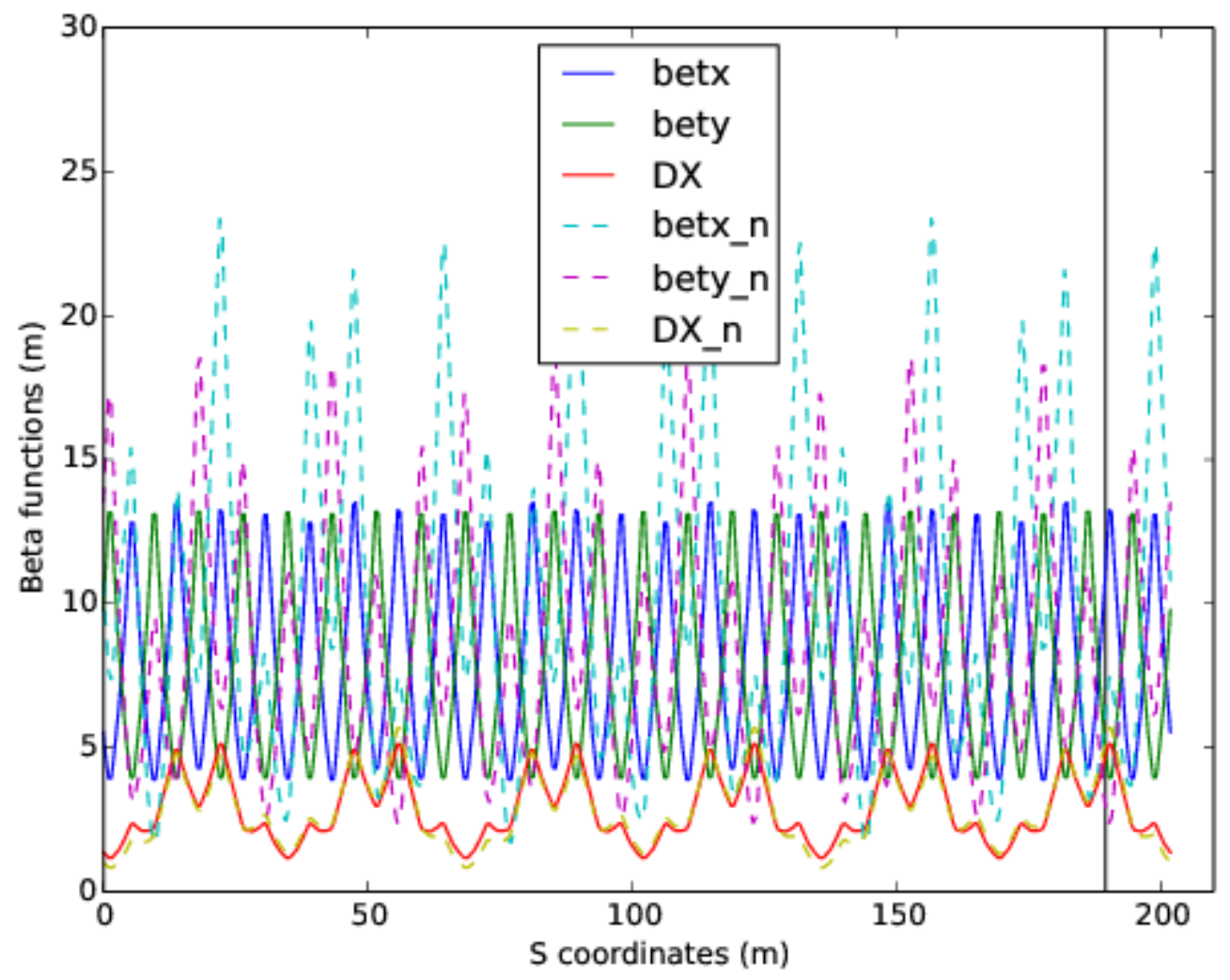

Figure 3: The distorted Twiss parameters in the Booster are shown in dashed lines, and the nominal ones are shown in the solid lines. The vertical black line is at the stripping foil.

\section{ALTERNATIVE SOLUTION WITH BETATRON TUNES BELOW HALF}

In this scheme, we will try to keep tunes below half for proton injection so that space charge would drag the betatron tunes away from half integer stopband. It also requires half integer crossing after injection which is a routine for NSRL operation, however not being demonstrated for RHIC operation [5]. The advantage of this scheme is that one may be able to reduce the beta functions at the stripping foil as needed with the half integer quadrupole in the available current range.

With betatron tunes being kept at $(4.44,4.47)$, we were able to reduce the beta functions at the foil down to $(6,4)$ $\mathrm{m}$ with available currents of the half integer quadrupoles. The currents are qvstr $1=-4.66$; qvstr $2=10.67$; qhstr $1=$ 13.44; qhstr2 $=19.12 ;$ iqhe $=-121.85 ;$ iqve $=-96.30$. The distorted Twiss functions around the Booster are shown in Figure 4. 


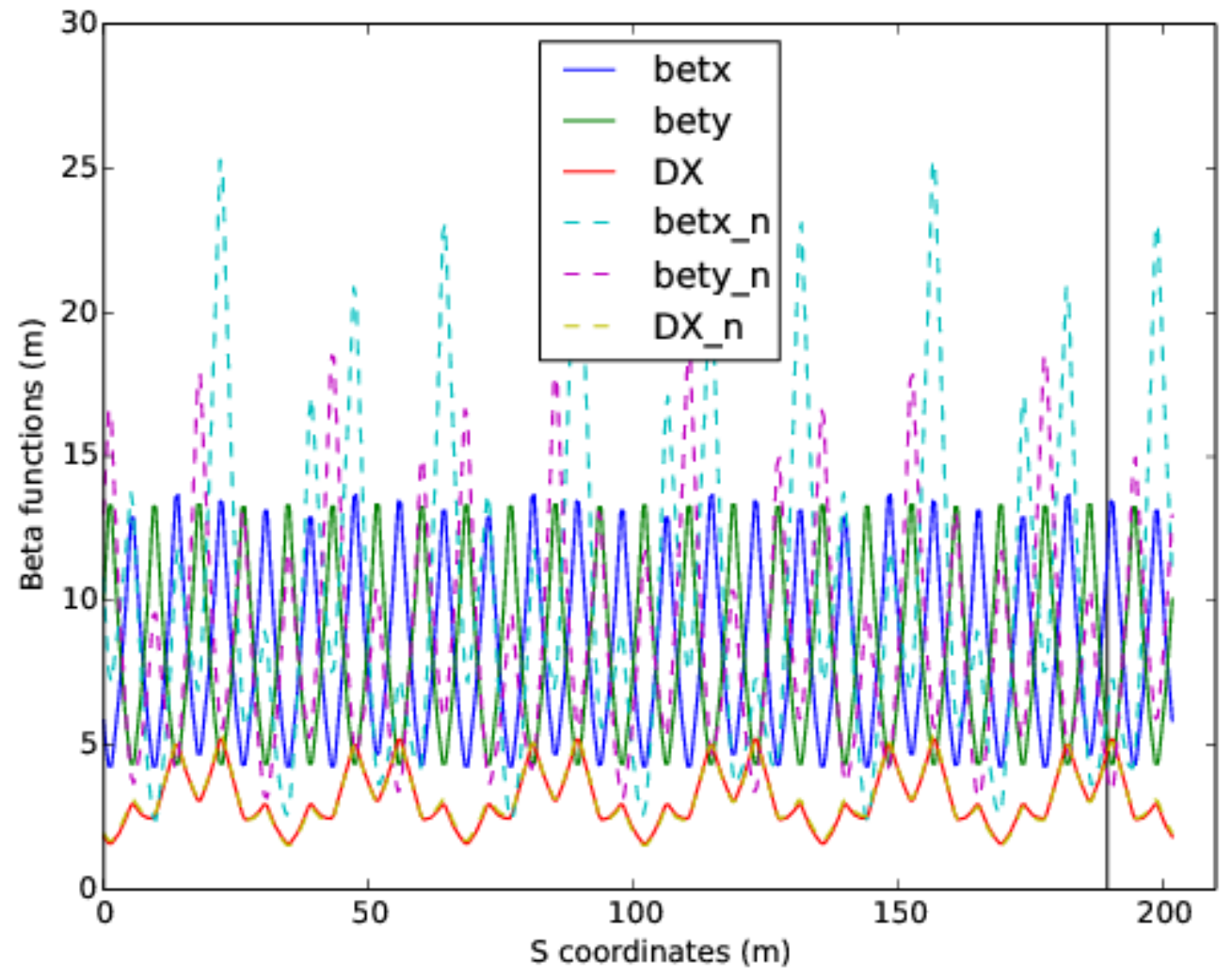

Figure 4: The distorted optics with tunes at $(4.44,4.47)$ is shown in dashed lines, and the nominal optics is shown in solid lines.

\section{SUMMARY}

The current injection optics in the Booster requires betatron tunes be close to and above half integer. As a result, the tune print of the proton beam may overlap with the half integer resonance due to the space charge force. We proposed two alternative optics which reduce beta functions at the foil. The first one which set the tunes at (4.7, 4.75) requires new power supplies for the half integer quadrupole strings. The second one which set the tunes at $(4.44,4.47)$ works within the current range of the half integer quadrupole strings. However, the beam has to cross half integer resonance after injection which is yet to be demonstrated for polarized proton.

\section{REFERENCE}

[1] Booster Design Manual, BNL (October, 1988)

[2] Gardner, C. "Booster Stopband Corrections.", Booster Tech. Note 217 (1993).

[3] Brown, K. A., et al. "Minimizing emittance growth during H- injection in the AGS booster.", Proceedings of PAC09, Vancouver, Canada (2009).

[4] Marneris, I., Private communication.

[5] Brown, K. A., Private communication. 